\title{
Tightening the constraints on the mass distribution of the Frontier Fields MACSJ0416.1-2403 with VLT/MUSE spectroscopy
}

\section{Benjamin Clément, Johan Richard, Guillaume Mahler, Vera Patrício, David Lagattuta and the MUSE Consortium}

Centre de Recherche Astrophysique de Lyon (CRAL), Observatoire de Lyon Université Lyon 1, 9 Avenue Charles André, 69561 Saint Genis Laval email: benjamin.clement@univ-lyon1.fr

\begin{abstract}
We have combined the performances of the VLT/MUSE spectrograph together with the power of gravitational lensing by Frontier Fields galaxy clusters to offer a unique magnified view of the distant universe. The large field of view over a wide optical wavelength domain enables redshift measurements of numerous lensed galaxies in the cluster core. Spectroscopicallyconfirmed multiple-imaged systems are further used as strong constraints to improve the cluster mass model. Here, we focus on the galaxy cluster MACSJ0416.1-2403 and compare the revised magnification map with results from previous analysis.
\end{abstract}

Keywords. gravitational lensing, galaxies: clusters: individual (MACSJ0416.1-2403), techniques: spectroscopic, (cosmology:) dark matter

\section{Introduction}

While the main ground-based optical multi-object spectrographs obtain spectra of a few tens of objects at a time, the panoramic integral-field ability of the new VLT/MUSE spectrograph increases that number by an order of magnitude. MUSE observations of HST deep fields yield a wealth of new spectroscopic identifications, revealing faint emission-line galaxies not detected in deep broad-band images (Bacon et al. (2015)). Its field of view of $1 \mathrm{x} 1$ square arc minutes over the full 4750-9350A wavelength domain is perfectly scaled to the geometry of galaxy clusters. Therefore, when combined with the power of gravitational lensing, MUSE enables redshift measurements of numerous lensed galaxies in the cluster core, and in particular of tens of multiple-imaged systems (Richard et al. (2015)). Together with extremely deep HST images from the Frontier Fields program, the deep MUSE spectroscopy of the cluster MACSJ0416.1-2403 presented in this work allows us to propose an improved model of the cluster mass distribution.

\section{Observations \& preliminary results}

As part of the Guaranteed Time allocated to the MUSE consortium, we have secured two hours of observations (PI: Richard, J.) of MACSJ0416.1-2403 under good seeing conditions (0.6"-0.7"). The data cover the northern part of this elongated cluster (Fig. 1, bottom) which includes 31 multiple-image systems out of the 71 identified in the deep HST images (Jauzac et al. (2014)). We measured the redshift of 14 new systems with MUSE and one from additional Keck/MOSFIRE follow-up. In addition to 10 redshifts already published in previous works, this pushes the total to 25 systems and 75 multiple 

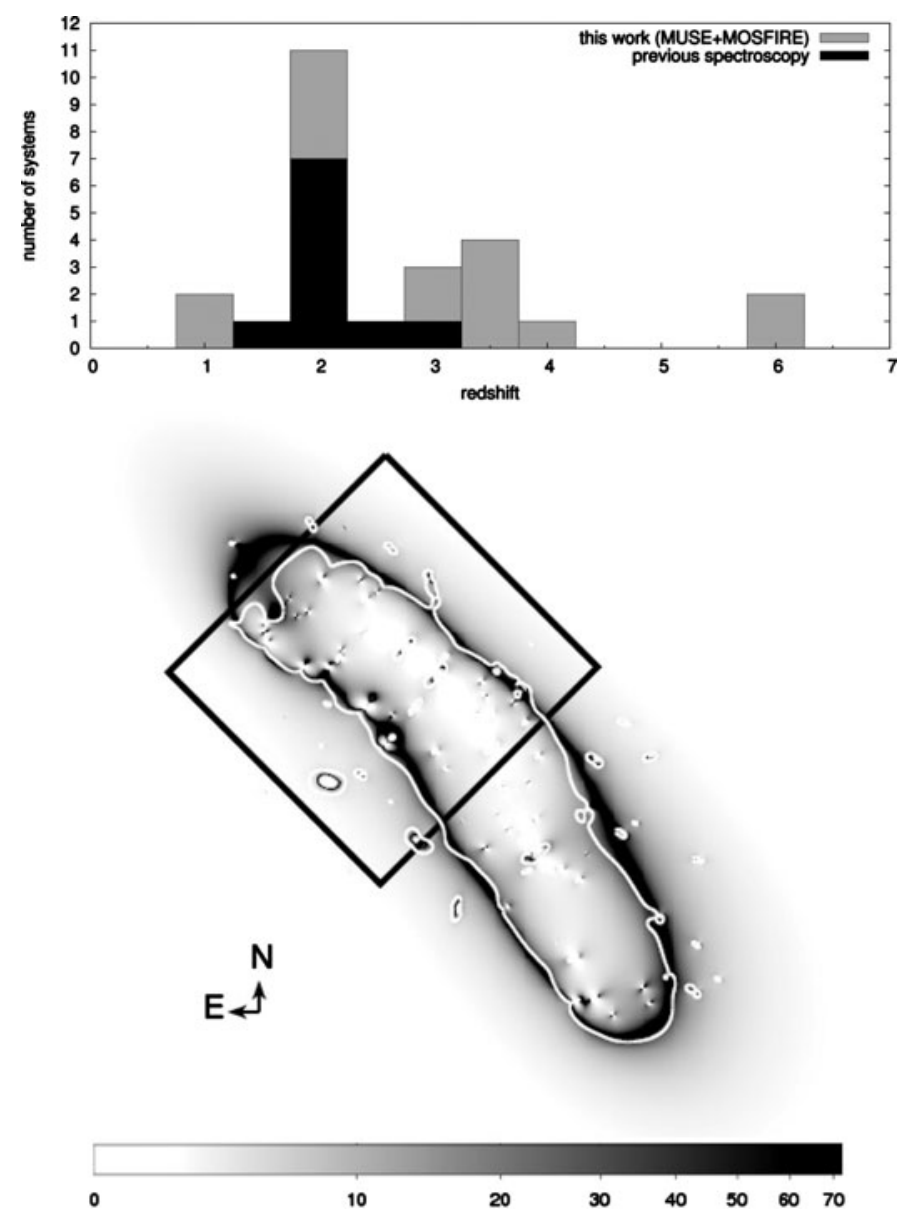

Figure 1. Top: distribution of the multiple-image system spectroscopic redshifts. Bottom: amplification map for a source at redshift $\mathrm{z}=6$. The grayscale image shows the absolute value of the amplification predicted by our best model. The white line is the critical line at $\mathrm{z}=6$ predicted by the HFFv2 model from the CATS team (Jauzac et al. (2014)). The black square shows the $1 \times 1$ arcmin footprint of the MUSE data.

images with spectroscopic redshifts (Fig. 1, top), making MACSJ0416.1-2403 the most well-constrained cluster to date. Only using systems with spectroscopic redshifts, our preliminary mass model predicts image positions with an rms of 0.46 ". Most notably, we revise the mass distribution (and the amplification) in the north-east part of the cluster where strong constraints were previously missing (Fig. 1, bottom).

\section{References}

Bacon, R., Brinchmann, J., Richard, J., Contini, T., Drake, A., et al. 2015, A $\& A$ A, 575A, 75B Jauzac, M., Clément, B., Limousin, M., Richard, J., et al. 2014, MNRAS, 443, 1549J

Richard, J., Patrício, V., Martinez, J., Bacon, R., Clément, B., et al. 2015, MNRAS, 446L, 16R 